\title{
NOUVELLE
}

\section{La boucle est bouclée}

Des enzymes qui détyrosinent les microtubules enfin découvertes

Marie-Jo Moutin, Christophe Bosc, Leticia Peris, Annie Andrieux

Grenoble Institut de Neurosciences, Inserm U1216, Université Grenoble Alpes, chemin Fortuné Ferrini, bâtiment Safra, 38700 La Tronche, France.

moutinm@univ-grenoble-alpes.fr
> Les microtubules sont des fibres du cytosquelette constituées par l'assemblage de dimères d' $\alpha$ - et de $\beta$-tubuline. Ces polymères en remodelage permanent jouent de multiples rôles dans les cellules. Organisés en fuseau, ils distribuent les chromosomes entre les cellules filles lors de la division cellulaire. Organisés en réseau, ils contribuent à la morphologie, la mobilité et la polarité des cellules, et constituent un réseau de rails pour le transport de divers constituants et organelles cellulaires. Pour assurer leurs nombreuses fonctions, les microtubules peuvent être très dynamiques ou stables. Cette plasticité dynamique est régulée de manière spatio-temporelle par plusieurs modifications post-traductionnelles que les microtubules

$\rightarrow$ Voir la Synthèse de K. Sadoul et al., page 1047 subissent $[1](\rightarrow)$.

La détyrosination, une modification réversible des microtubules connue de longue date

La détyrosination est la première étape d'une modification post-traductionnelle spécifique à la tubuline généralement nommée cycle de détyrosination/tyrosination. Ce cycle, découvert par une équipe argentine il y a 40 ans (en 1977) [2], fait intervenir deux enzymes. La première enzyme qui modifie la tubuline est l'enzyme de détyrosination, ou tubuline carboxypeptidase (TCP): elle clive la tyrosine présente au C-terminus de la plupart des isoformes de tubuline $\alpha^{1}$. Une deuxième enzyme, la tubuline tyrosine ligase (TTL), inverse l'action de la TCP en rétablissant cette tyrosine. Le cycle module ainsi dans les cellules la présence de deux formes de tubuline $\alpha$, détyrosinée et tyrosinée, dont les propriétés physicochimiques et les partenaires protéiques cellulaires diffèrent. II conduit également à la genèse d'autres formes de tubuline $\alpha$ tronquées à l'extrémité C-terminale dont le rôle reste encore très peu étudié $[3,4]$. Le cycle est intimement lié à la dynamique des microtubules. Les microtubules nouvellement polymérisés sont majoritairement tyrosinés (la TTL agissant uniquement sur le dimère soluble de tubuline $\alpha / \beta$ ), alors que les microtubules stables sont préférentiellement détyrosinés. De plus, la détyrosination renforce la stabilisation des microtubules en inhibant le recrutement de moteurs moléculaires dépolymérisant, comme la kinésine 13 [5].

L'identification de la TTL en 1993 et les études qui en ont découlées, en particulier l'analyse des cellules et des souris déficientes pour cette enzyme, ont révélé le rôle vital de ce cycle pour la cellule et pour l'organisme [6]. En revanche, bien que la réaction de détyrosination soit connue depuis 1977 [7], le(les) acteur(s) cellulaire(s) en charge de cette activité restai(en)t à découvrir.

${ }^{1}$ Les isoformes de tubuline $\alpha 1 \mathrm{~A}, \alpha 1 \mathrm{~B}, \alpha 1 \mathrm{C}, \alpha 3 \mathrm{~A}$, et $\alpha 3 \mathrm{~B}$ portent une tyrosine, $\alpha 8$ une phénylalanine et $\alpha 4$ est néosynthétisée directement sans tyrosine dès sa néosynthèse.
Une méthode originale, une découverte inattendue

À partir des années 1980, plusieurs purifications biochimiques partielles de l'enzyme de détyrosination ont été reportées, toutes réalisées à partir de tissu cérébral dans lequel l'activité s'était révélée abondante. Nous nous sommes appuyés sur ces travaux pour concevoir une procédure de purification biochimique en trois étapes qui nous a permis d'enrichir 400 fois l'activité de détyrosination dans des homogénats de cerveaux (Figure 1A) [8]. L'activité enzymatique a été suivie lors des différentes étapes de purification par un test utilisant des microtubules radio-marqués comme substrat. Une tyrosine marquée au ${ }^{14} \mathrm{C}$ a été additionnée sur la tubuline $\alpha$, puis les dimères $\alpha / \beta$ ont été polymérisés pour former des microtubules, stabilisés par du taxol. Utiliser des microtubules comme substrats permettait de s'assurer de la fiabilité du test, puisque plusieurs travaux avaient montré que la TCP préférait les microtubules à la tubuline non polymérisée.

Pour isoler la ou les protéine(s) responsable(s) de l'activité de détyrosination dans la fraction enrichie, nous avons utilisé un inhibiteur irréversible comme hameçon dans une stratégie de chimie click. La chimie click consiste à associer deux molécules l'une à l'autre par une réaction très maîtrisée, le plus souvent entre des groupements alcyne et azide. Nous avons commencé par déterminer la famille enzymatique de la TCP cérébrale en étudiant sa sensibilité à 


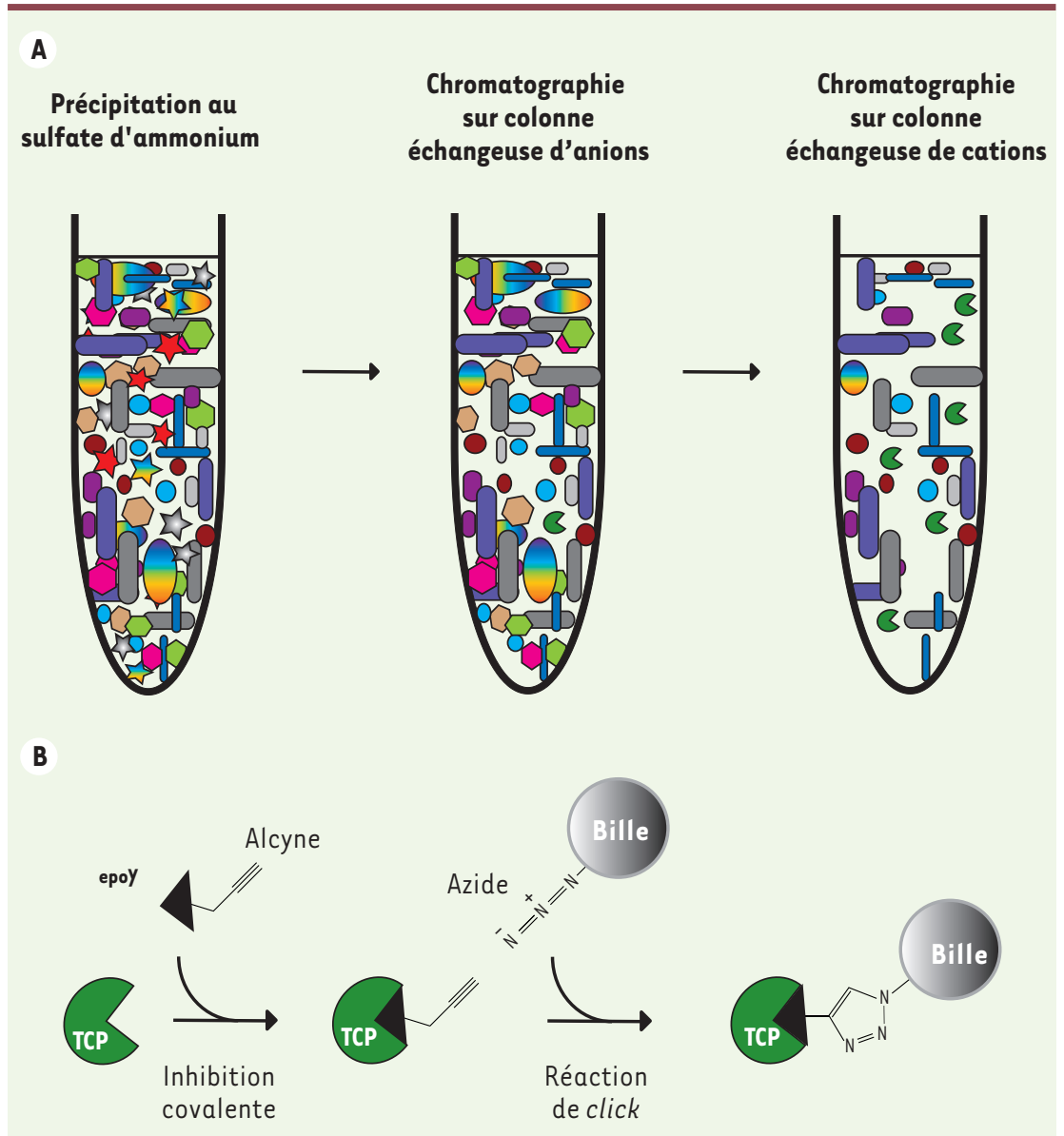

différents inhibiteurs de protéases et en avons déduit que son activité catalytique dépendait d'une cystéine réactive. L'équipe de Matthew Bogyo, de I'université de Stanford, a alors développé plusieurs inhibiteurs mimant la partie C-terminale de la tubuline et comprenant un groupement époxyde capable - s'il est correctement orienté dans la poche catalytique - de se fixer irréversiblement sur la cystéine réactive de l'enzyme. Plusieurs molécules ont été testées et la plus simple, epoy (contenant seulement la tyrosine de l'extrémité C-terminale de la tubuline), a permis de «capturer » l'enzyme parmi d'autres protéines (Figure 1). Pour cela, un groupement alcyne a été additionné sur l'inhibiteur liant irréversiblement l'enzyme, ce qui permet sa fixation sur des billes d'agarose portant un groupement azide (Figure 1B). Après analyse par spectrométrie de masse des protéines immobilisées sur les billes, un seul
Figure 1. Représentation schématique des étapes de purification de l'enzyme de détyrosination (TCP). Trois étapes d'enrichissement (A) suivie d'une dernière étape d'affinité fondée sur l'utilisation d'un inhibiteur irréversible (epoy) et une réaction de chimie click (une cyclo-addition d'un azide sur un azoture catalysée par le cuivre) (B) ont permis d'aboutir à l'identification de l'enzyme majoritaire dans le cerveau.

sur l'angiogenèse et/ou en cancérologie, essentiellement par l'équipe de Yasufumi Sato au Japon [10]. Alors que la vasohibine- 1 est anti-angiogénique, la vasohibine-2 semble pro-angiogénique. Les deux protéines sont enrichies dans plusieurs cancers et sont des facteurs de mauvais pronostic. Les vasohibines sont des protéines de 41-42 kDa qui se lient avec une très forte affinité à une protéine nommée SVBP (small vasohibin-binding protein), considérée comme une protéine chaperonne nécessaire à leur stabilité et leur sécrétion [11]. Nos résultats de protéomique, associés aux données de la littérature, nous ont conduits à examiner la capacité des vasohibines à détyrosiner la tubuline $\alpha$ en l'absence ou en présence de SVBP. Nous avons montré que les complexes vasohibine-1/SVBP et vasohibine-2/SVBP ont une activité tubuline carboxypeptidase (TCP) dans les cellules et dans les tests réalisés in vitro avec les complexes purifiés: une tyrosine ou une phénylalanine peut être clivée à l'extrémité de la tubuline $\alpha$ par ces complexes, générant des formes détyrosinées de la tubuline. La cystéine prédite catalytique est indispensable: sa mutation abolit l'activité TCP. Et, comme attendu, les complexes enzymatiques ont une nette préférence pour les microtubules comparativement aux dimères libres de tubuline $\alpha / \beta$.

\section{Les complexes vasohibines/SVBP} régulent la différenciation neuronale Nous avons ensuite examiné l'importance physiologique des complexes enzyma- 


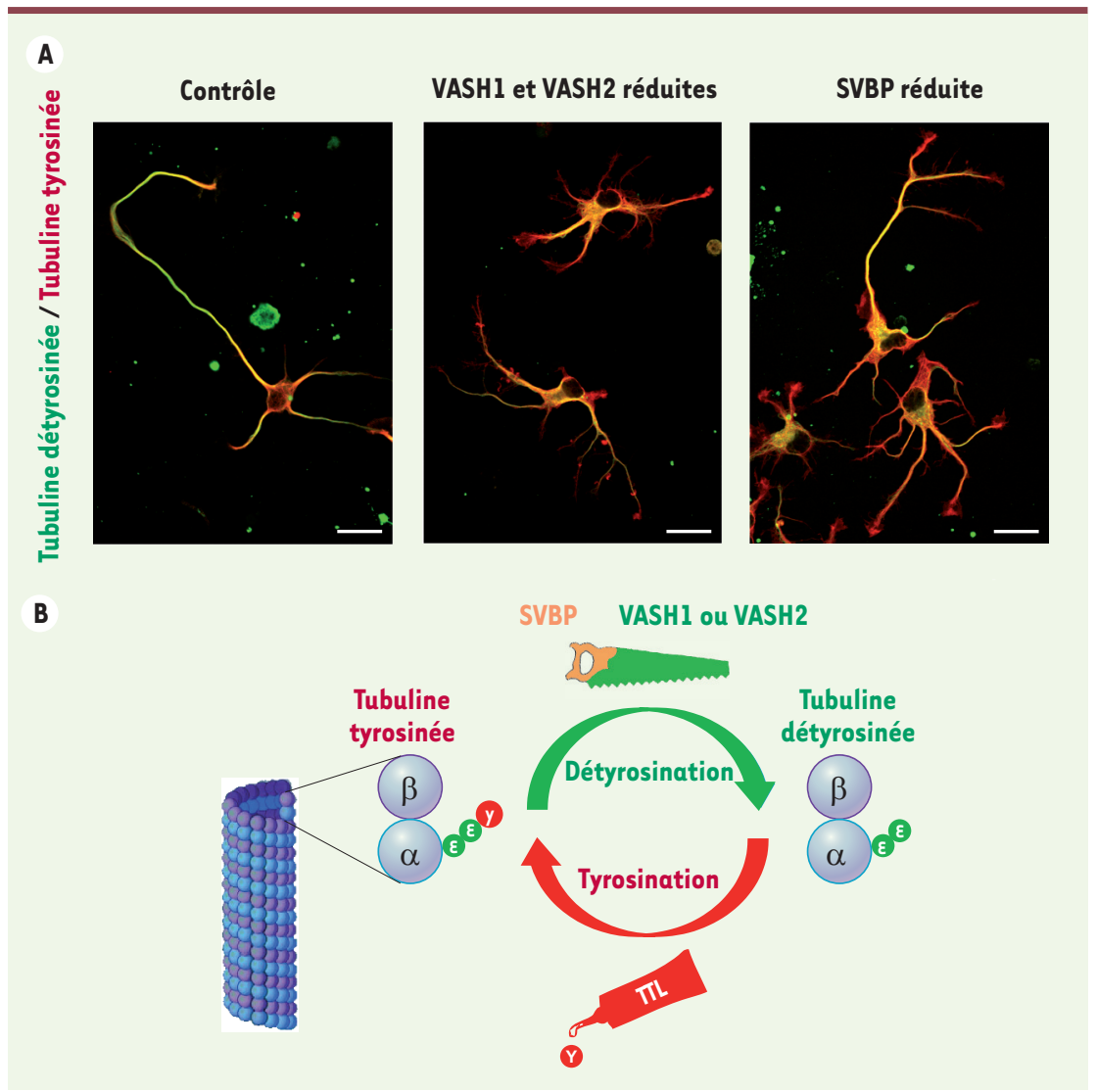

Figure 2. La détyrosination de la tubuline est essentielle au développement des neurones. A. Altération des neurones lors d'une réduction de l'expression des complexes enzymatiques vasohibine/SVBP (small vasohibin-binding protein). De gauche à droite: neurone contrôle, neurones dans lesquels vasohibine-1 (VASH1) et vasohibine-2 (VASH2) ont été réduites, et neurones dans lesquels SVBP a été réduite. Les neurones exprimant moins d'enzymes présentent un retard de développement et des anomalies morphologiques. B. Cycle de détyrosination/ tyrosination de la tubuline. Les microtubules sont composés d'un empilement de dimères de tubulines $\alpha / \beta$. La tubuline $\alpha$ porte une tyrosine $(y)$ à son extrémité. Les enzymes de détyrosination (tubuline carboxypeptidases, TCP) représentées par une scie composée des éléments vasohibines (VASH 1,2) et SVBP clivent la tyrosine sur les microtubules. La TTL représentée par un tube de colle replace la tyrosine sur le dimère libre de la tubuline. La surface des microtubules est ainsi tyrosinée ou détyrosinée. Ce cycle, lié à la dynamique des microtubules, est essentiel à diverses fonctions des microtubules dans les cellules (division, migration, etc.) et vital pour l'organisme.

tiques de détyrosination en nous focalisant sur les neurones et le cerveau pour lesquels le cycle de détyrosination/tyrosination s'était révélé fondamental. Dans les neurones, la réduction de l'expression des deux vasohibines, ou de SVBP, par des ARN interférents induit des défauts morphologiques (une augmentation du nombre des neurites et des branchements neuritiques) et ralentit la différenciation axonale (Figure $2 A$ ). Des résultats équiva- interférents et de l'inhibiteur ne conduit pas à une réduction totale de la détyrosination dans les neurones, laissant suspecter la présence d'autres enzymes de détyrosination dans ces cellules. La présence de tubuline $\alpha 4$, qui n'a pas de tyrosine $\mathrm{C}$-terminale, pourrait également contribuer à ce résultat. Les complexes enzymatiques vasohibine/SVBP sont de plus impliqués dans le développement du système nerveux central puisque la diminution de leur expression dans le cortex cérébral d'embryon de souris affecte la migration radiale de ces neurones.

\section{Perspectives}

La méconnaissance des enzymes portant l'activité de détyrosination a longtemps bloqué la compréhension du cycle de détyrosination/tyrosination de la tubuline. Nous avons récemment découvert l'activité de détyrosination des complexes de vasohibine/SVBP pour la détyrosination de la tubuline (Figure 2B), ce qui a été simultanément confirmé par une équipe hollandaise utilisant une approche génétique [12]. Un test réalisé sur des cellules haploïdes (ne possédant qu'un seul exemplaire de chaque chromosome et donc de la plupart des gènes) leur a en effet permis de révéler l'importance du gène SVBP pour la détyrosination de la tubuline, puis d'étudier les complexes formés avec les vasohibines. Ainsi, cette énigme de 40 ans vient donc d'être résolue par deux équipes européennes. C'est très probablement parce qu'il s'agissait de complexes enzymatiques que leur révélation a été si compliquée et si longue, nécessitant des approches inédites.

L'identification de ces enzymes devrait permettre de mieux comprendre les liens entre détyrosination et dynamique microtubulaire, et comment les cellules utilisent la détyrosination pour contrôler certaines fonctions des microtubules. II s'agira donc de déterminer dans quels tissus les complexes enzymatiques vasohibine/SVBP jouent un rôle majeur, d'évaluer la spécificité de chacun d'entre eux (tissulaire, cellulaire ou subcellulaire), 
et de décrire comment leur activité est régulée puis relayée dans les cellules pour des fonctions microtubulaires dépendant de leur détyrosination. La génération d'animaux déficients pour ces protéines sera d'un grand intérêt pour aborder ces différentes questions. Les futures études in vitro (structure-fonction) apporteront, quant à elles, des réponses aux questions d'ordre moléculaire: comment se lie la vasohibine à la protéine chaperonne SVBP ? Comment interagissent ces complexes enzymatiques avec la tubuline $\alpha$ des microtubules ? Ces enzymes sontelles processives (c'est-à-dire capable de catalyser des réactions successives sur un microtubule sans le relâcher)?

D'autres questions intéressantes sont soulevées par ces travaux. Les vasohibines sont sécrétées par les cellules épithéliales [11]. Qu'en est-il des autres types cellulaires? Quel rôle jouent les vasohibines à l'extérieur des cellules? La protéine chaperonne SVBP étant sécrétée simultanément, ces protéines sont-elles actives dans le stroma et sur quel(s) substrat(s)?

Les nombreuses questions relatives aux complexes vasohibine/SVBP devraient trouver des réponses dans des délais plus raisonnables que celui qui a été nécessaire à l'identification de leur fonction enzymatique. $\diamond$

After 40 years of mystery, the enzymatic complexes that detyrosinate microtubules finally identified

\section{LIENS D'INTÉRÊT}

Les auteurs déclarent n'avoir aucun lien d'intérêt concernant les données publiées dans cet article.

\section{RÉFÉRENCES}

1. Sadoul K, Joubert C, Michallet S, et al. Sur la voie du déchiffrage du code tubuline : le point sur l'acétylation et la détyrosination. Med Sci (Paris) 2018 ; 34 : 1047-55.

2. Barra HS, Arce CA, Argarana CE. Posttranslational tyrosination/detyrosination of tubulin. Mol Neurobiol $1988 ; 2: 133-53$.
3. Aillaud C, Bosc C, Saoudi Y, et al. Evidence for new C-terminally truncated variants of alpha- and betatubulins. Mol Biol Cell $2016 ; 27: 640-53$.

4. Lafanechere L, Job D. The third tubulin pool. Neurochem Res $2000 ; 25: 11-8$.

5. Peris L, Wagenbach M, Lafanechere L, et al. Motordependent microtubule disassembly driven by tubulin tyrosination. J Cell Biol 2009 ; 185 : 1159-66.

6. Erck C, Peris L, Andrieux A, et al. A vital role of tubulintyrosine-ligase for neuronal organization. Proc Natl Acad Sci USA 2005 ; 102 : 7853-8.

7. Hallak ME, Rodriguez JA, Barra HS, et al. Release of tyrosine from tyrosinated tubulin. Some common factors that affect this process and the assembly of tubulin. FEBS Lett $1977 ; 73$ : 147-50.

8. Aillaud C, Bosc C, Peris L, et al. Vasohibins/SVBP are tubulin carboxypeptidases (TCPs) that regulate neuron differentiation. Science 2017 ; 358 : 1448-53.

9. Sanchez-Pulido L, Ponting CP. Vasohibins: new transglutaminase-like cysteine proteases possessing a non-canonical Cys-His-Ser catalytic triad. Bioinformatics 2016 ; 32 : 1441-5.

10. Sato $Y$. The vasohibin family: a novel family for angiogenesis regulation. J Biochem 2013 ; 153 : 5-11.

11. Suzuki $Y$, Kobayashi M, Miyashita H, et al. Isolation of a small vasohibin-binding protein (SVBP) and its role in vasohibin secretion. J Cell Sci 2010 ; 123 : 3094-101.

12. Nieuwenhuis J, Adamopoulos $A$, Bleijerveld $O B$, et al. Vasohibins encode tubulin detyrosinating activity. Science 2017 ; 358 : 1453-6.

\section{NOUVELLE}

Transition des cellules de
mélanome vers un phénotype
invasif et pharmacorésistant
Un rôle pour NAMPT et le NAD
Corine Bertolotto, Mickaël Ohanna, Robert Ballotti

> Le mélanome cutané dérive des mélanocytes, des cellules de la peau dont la fonction physiologique est la production de pigments mélaniques responsables de notre couleur de peau en lien avec la protection solaire. Son incidence a augmenté ces trois dernières décennies et c'est environ 15000 nouveaux cas qui sont diagnostiqués chaque année en France. Dépisté de manière précoce, le mélanome se guérit, avec $85 \%$ de survie sans récidive à 5 ans. En revanche, s'il devient métastatique, le taux de survie à 5 ans chute dramatiquement à 10-15\%. Des avancées spectaculaires ont été réalisées récemment dans le traitement du mélanome cutané métastatique grâce aux thérapies ciblées et aux immunothérapies qui, pour la première fois, ont permis d'augmenter la survie globale des patients. Cependant, il reste un besoin thérapeutique important pour cette maladie, car les réponses cliniques restent transitoires ou limitées à des sous-groupes restreints de patients
Université Nice Côte d'Azur, Inserm, C3M, 151, route Saint Antoine de Ginestière, 06204 Nice, France; Inserm, U1065, Biology and pathologies of melanocytes. Équipe labellisée ARC 2015, Nice, France.

bertolot@unice.fr

en raison de résistances intrinsèques ou acquises. Ces résistances peuvent avoir pour origine des altérations génétiques, mais la plasticité phénotypique due aux remodelages génique et métabolique de la tumeur est maintenant acceptée comme la principale source de résistance [1]. Les mélanomes sont constitués de deux sous-populations cellulaires principales: les cellules prolifératives et invasives, qui, sous l'effet de stress (I'hypoxie, la carence en nutriments, le traitement lui-même, 